\title{
Single-Stage Polystyrene Concrete 3D Printing Technology
}

\author{
Victor Molodin ${ }^{1, *}$, Ivan Gasenko ${ }^{2}$, and Pavel Timin ${ }^{3}$ \\ ${ }^{1}$ Doctor of Technical Sciences, Professor, Head of the Department of Construction Technology and \\ Organization at Novosibirsk State University Architectural and Civil engineering (Sibstrin), \\ Novosibirsk, 630008, Russian Federation \\ ${ }^{2}$ Student of the Institute of Civil Engineering, at Novosibirsk State University Architectural and Civil \\ engineering (Sibstrin), Novosibirsk, 630008, Russian Federation \\ ${ }^{3}$ Student of the Institute of Civil Engineering, at Novosibirsk State University Architectural and Civil \\ engineering (Sibstrin), Novosibirsk, 630008, Russian Federation
}

\begin{abstract}
The 3-D printers offered to the construction market can perform retained form layer-by-layer for subsequent filling with reinforced structural concrete and insulation. The use of a single-stage polystyrene concrete with polystyrene granules bloated directly in the printer head allows to form layer-by-layer the insulated wall for its entire width and reduce the erection operation to one technological conversion.
\end{abstract}

\section{Introduction}

Within the implementation framework of the National Project "Housing and Urban Habitat", the builders of Russia have serious tasks to increase the commissioning of housing and social infrastructures. In 2019, only in the Siberian Federal District about 7 million sq. $\mathrm{m}$ of housing was commissioned that is $8.75 \%$ of the housing commissioning in Russia. The planned figures for 2020 are even higher. They are 8 million 400 thousand sq. $\mathrm{m}$ that is the increase should be up to $21 \%$ of the achieved result. It is a difficult task, and to solve it, we have to mobilize all available resources, and introduce new technologies and materials widely.

One of the perspectives for development of modern construction is additive technologies that are layer-by-layer growth and synthesis of three-dimensional objects by adding material to the base. The first proposal to use 3D printing for the needs of the construction industry is recorded by a patent issued in the United States to Behrokh Khoshnevis in 2004 [1]. The proposed robotic system was a portal printer with a working head in the form of an extruder (Fig.1) that firstly extrudes layer-by-layer two side walls out of structural concrete, and then, during the second run, fills the cavity between them with heat insulating or other binding material (Fig. 2).

\footnotetext{
* Corresponding author: molodin@sibstrin.ru
} 


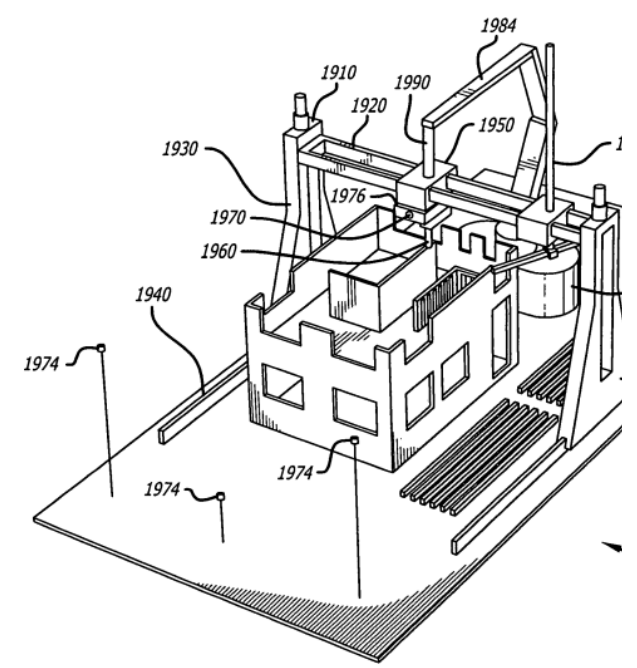

Fig.1. General view of the portal 3D printer printer working hea on the USA patent US7641461B2

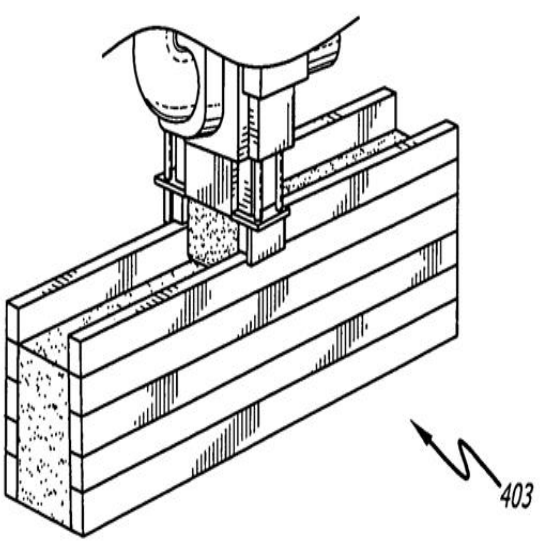

Fig.2. General view of the portal 3D on the USA patent US7641461B2.

The proposed technical solution has been the basic for long 12 years. It has been extensively improved both in terms of the printer itself and in terms of the material to be extruded $[2,3,4,5,6]$. During this period, more than 300 patents have been issued that improved the system. Since 2014 the technology has experimentally reached the construction sites. The Chinese company "WinSun" moved from semi-reproduction experiments to the printing of real one-storey and two-storey houses [7], using a bulky portal printer. The attempts to overcome the shortcomings of the predecessors have been made by "DCP" (USA) that offered to the market a hydraulic crane with a single-stage manipulator with six degrees of freedom and a set of working heads, including extruders for work with concrete, thermoplastic, soil and foamed polyurethane.

In 2016, a radial 3D construction printer, the development of "Apis Cor", appeared on the market [8]. It allowed simplifying the structure greatly while increasing the technological capabilities of the device. The application experience showed the advantages of a radial printer over a portal printer, but the extrusion technology - firstly layer-by-layer extrusion of the side walls and then filling the cavity between them - remained unchanged. Approximately at the same time, the 3D printer forming sidewalls not from cement-sand mixture, but from polyurethane foam has been developed at the University of Nantes together with the "Nantes Digital Sciences Laboratory (LS2N)" [9]. Then the cavity between the walls is filled with structural concrete. Such a wall has a structural part inside and insulation from outside. The use of polyurethane foam allowed accelerating the process of rigid side walls formation of the structure and at the same time to insulate them. However, the high cost of the materials and polyurethane surface of the wall, that require a lot of additional expenses for their protection from sunlight and finishing, make the project of little promise despite its attractiveness.

In recent years, there have been some attempts at the construction market to implement 3D technologies using clay to make wall blocks [10], sand that is impregnated with adhesive solution [11]. However, there is no serious market promoting of 3D printer yet.

It should be noted that all attempts to improve the 3D printer and extruding binders in recent years have not changed the fundamental solution of the basic patent [1]. First, the 
composition made of cement, clay or polyurethane form the wall frame or a retained form, and then the formed cavities are filled with insulation or structural concrete (fig.3)

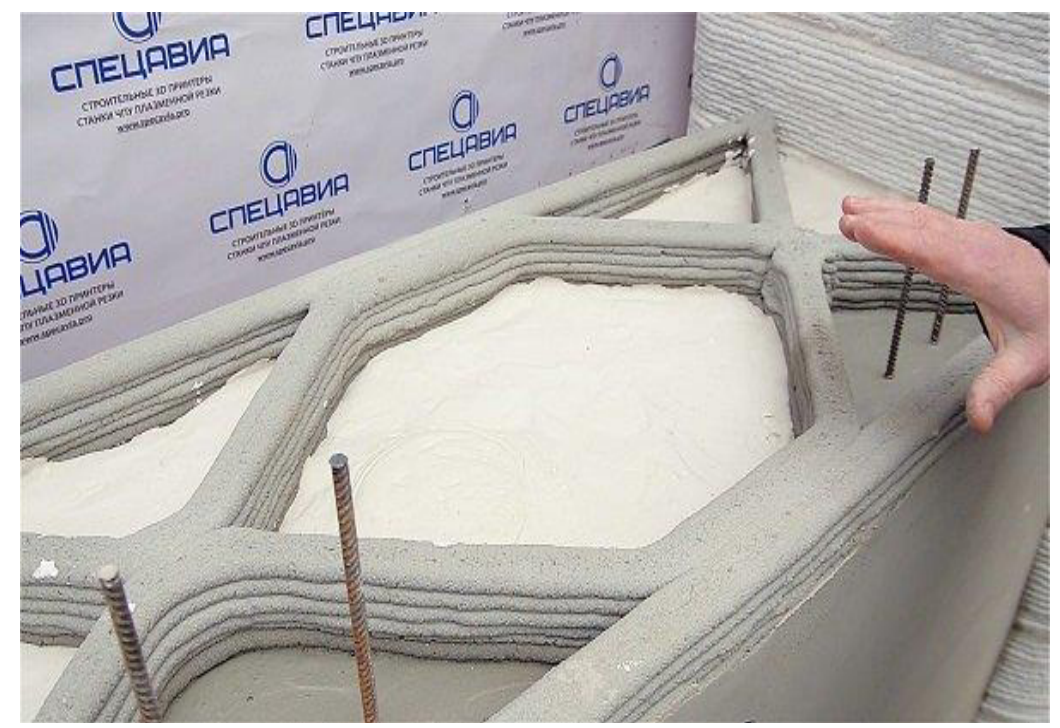

Fig. 2. A wall structure made with a 3D printer according to the traditional technology.

The developed technology has a number of disadvantages that delay its wide implementation into the construction.

1. A high labour intensity of the process to get a final product. First, a wall frame or a retained form is formed with a single binder by the $3 \mathrm{D}$ printer. Then, having waited for the loss of mobility of the extruded material, the formed cavities are partially reinforced and then filled with structural concrete and partially filled with insulation. It should be noted that all operations except the printing of a retained form are performed manually.

2.It is possible to combine the wall frame printing and a simultaneous filling of the formed cavity with light concrete [1]. At the same time, the structure of the printing head and its operation are considerably complicated by the need to include into the structure two systems to supply different binding materials.

3. The necessity for sufficiently rapid curing of the wall frame binder to carry the weight of the superimposed binder layers and the lateral pressure of the binder that fill the cavities. At the same time it should not cure too quickly because it can lead to its curing within material storage and delivery systems.

4.In order to avoid the bleeding of the lower, previously extruded layers under the weight of the upper layers, it is necessary to make a technological pause that significantly complicates the construction process and increases its duration.

5.A successful operation of a construction 3D printer using cement-based binder requires positive temperatures or a greenhouse arrangement during the cold season.

The objective of the present work is to develop additive technology to manufacture the enclosing structures of buildings that will allow to overcome disadvantages mentioned above and will be able to provide automated (without people) construction of insulated walls with a minimum number of technological changes.

The task of the present research is to study the workability of a single-stage polystyrene concrete as a binder. 


\section{Materials and methods}

Figures and tables, as originals of good quality and well contrasted, are to be in their final form, ready for reproduction, pasted in the appropriate place in the text. Try to ensure that the size of the text in your figures is approximately the same size as the main text $(10$ point). Try to ensure that lines are no thinner than 0.25 point. The single-stage polystyrene concrete technology developed in NSUACE (Sibstrin) allows significantly simplifying the process of building enclosing structures using the $3 \mathrm{D}$ printer [12]. The essence of the technology is that granules of non-foaming (beaded) polystyrene are added into the mixture of cement and sand during its processing.

The density of the beaded polystyrene coordinates the density of the cement and sand mixture. Therefore, while mixing the polystyrene granules are uniformly distributed throughout the mass of the material. The ready mixture is placed in the space between plate electrodes and the current of industrial frequency, of $220 \div 380 \mathrm{~V}$ is applied to the plates. In the course of the forced electro heating the temperature of the mixture quickly rises up to $80^{\circ} \mathrm{C}$, at such a temperature, the granules of beaded polystyrene bloats in many times, increasing in volume. At the same time, if the mixture is in a closed space, by increasing the volume of polystyrene granules during the foaming, self-consolidation of the material takes place. Changing the amount of polystyrene in the mixture, the structural or heatinsulating properties of the material can be enhanced.

All the advantages of single-stage polystyrene are realized for additive technology of construction with insulated walls. The technology is implemented as follows (Fig. 3). Polystyrene concrete mixture with non-foaming granules of beaded polystyrene 8 through the feeding system of the binder 2 enters the extruder 1
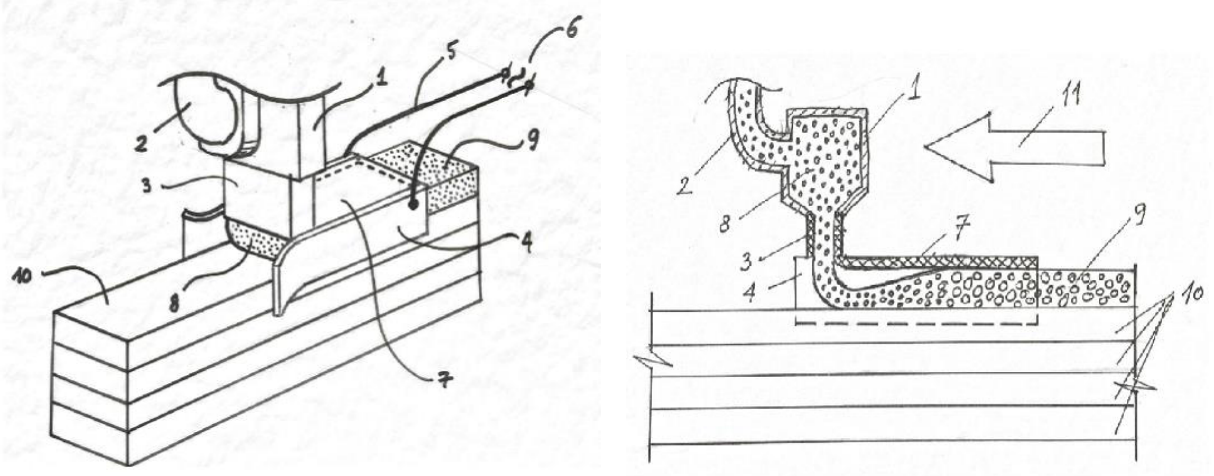

Fig. 3. Principal solution of $3 \mathrm{D}$ printer working head for extrusion of polystyrene concrete mixture that is laid according to one-stage technology.

and is heated there to the temperature close to the temperature of polystyrene foaming but it does not reach it. From that place, through the discharge outlet (nozzle) 3, the mixture is pressed onto the surface of the previously molded and solid layer of polystyrene concrete 10. The nozzle 3 has got a transverse dimension equal to the width of the erected wall 10 . The polystyrene concrete mixture 8 fills the space between the spatula electrodes 4 those are being the part of the printing head of the 3D printer, move slowly and continuously 11 along the wall.

The electric current $220 \div 380 \mathrm{~V}$ that is coming from the electrical network 6 through the cables 5 , passes through the polystyrene concrete mixture and heats it quickly. When the temperature reaches $80^{\circ} \mathrm{C}$, the polystyrene granules soften, and under the influence of the boiling isopentane contained therein, the granules foamed and increased in volume many 
times over, changing the quality of the 9 mixture. While heating and polystyrene granules bloating, the volume of polystyrene concrete mixture 9 increases and occupies the whole space between spatula electrodes 4 , the surface of previously laid layer 10 and pressing spatula 7, partially consolidates. Spatula electrodes 4 and pressure spatula 7 form another layer of the wall from polystyrene concrete 9 that was the result of electric thermal treatment of heat-insulating properties. Having a high temperature, polystyrene concrete begins to lose mobility very quickly and harden intensively still being in the space between the spatula electrodes. Having been uniformly distributed in volume the foamed granules of polystyrene, which are insulation as well, reduce heat loss from the structure into the environment.

The duration of maintaining high temperatures inside the material is increased; thereby it provides an intense increase in the strength of the polystyrene concrete. Changing the content of polystyrene in the binder formulation as required, it is possible to increase or decrease the thermal insulation or structural properties of the wall.

The full-scale testing of the 3D printer head for extrusion of polystyrene concrete mixture (fig. 4) that is laid according to one-stage technology showed that the working layer of the material formed according to the proposed technology retains cohesive and shows no signs of mobility. Laying the next working layers on the first one in 15 minutes after the first run of $3 \mathrm{D}$ printer does not practically affect the acquired shape and geometric dimensions of the first layer.

a.

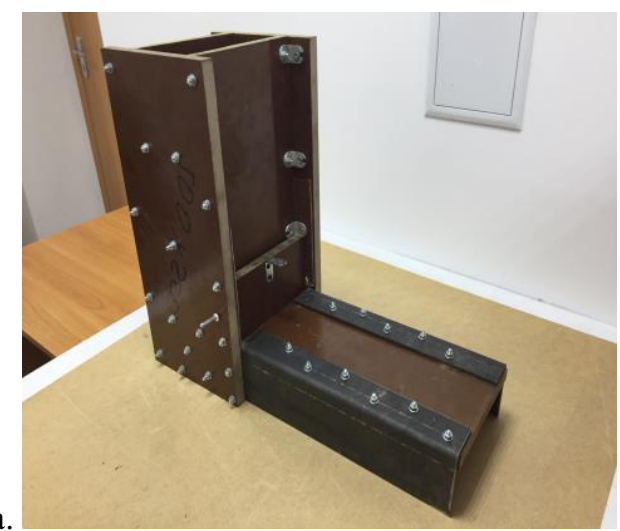

b.

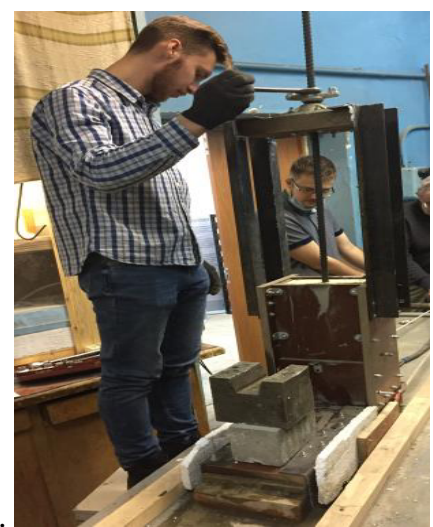

Fig. 4. General view (a) and full-sale testing (b) of 3D printer working head for printing the enclosing structures with single-stage polystyrene concrete.

The obtained polystyrene concrete samples were tested for strength and distribution uniformity of the foamed polystyrene granules by the weight of the material.

The cube-strength tests of the samples were performed at 3, 7, 14 and 28 day period. Three standard samples were cut for the tests. Table 1 shows the average test values. The tests were carried out on a standard laboratory press (PSU-50).

Table 1 Dynamics of polystyrene concrete strength developing

\begin{tabular}{|c|c|c|c|c|}
\hline Type of curing & $\begin{array}{l}3 \text { days, } \\
\mathrm{R}, \mathrm{MPa}\end{array}$ & $\begin{array}{l}7 \text { days, } \\
\mathrm{R}, \mathrm{MPa}\end{array}$ & $\begin{array}{l}14 \text { days, } \\
\text { R, MPa }\end{array}$ & $\begin{array}{l}28 \text { days, } \\
\mathrm{R}, \mathrm{MPa}\end{array}$ \\
\hline Electro heating, D1200kg/m ${ }^{3}$ & 3.83 & 7.71 & 9.38 & 12.73 \\
\hline Electro heating, D700 kg/m & 2.72 & 5.05 & 5.87 & 7.46 \\
\hline Standard technology,D1200 kg/m & 3.21 & 5.33 & 7.10 & 10.69 \\
\hline
\end{tabular}


Analyzing the test results of the polystyrene concrete strength of the medium density D700 and D1200 after the heat treatment, it can be stated that the strength of the samples made according to the one-stage technology is higher than that of the natural hardening samples. The structure of the obtained material proved to be good. The beaded polystyrene foamed entirely and over the whole volume. The average strength compared to polystyrene of standard technology was $20 \%$ higher.

The uniformity of polystyrene granules distribution by the material mass was determined by means of measuring of flat samples thermal conductivity cut from the wall mass by sections perpendicular to the direction of possible surfacing of granules.

Before measuring the thermal conductivity, the samples were dried to a constant mass in the oven at $70^{\circ} \mathrm{C}$ within 24 hours.

The weight loss of the samples was about 5\%. The geometric characteristics of the sample, the electrical power required to create heat current and the temperature of the opposite faces sides were measured.

In the course of the experiment, by means of thermal conductivity measuring device (PIT-2), the thermal conductivity of samples cut from a wall made of medium density polystyrene D700 and D1200, a working head of the 3D printer according to one-stage technology, as well as the sample made according to a standard technology of medium density D1200 (Table 2) were determined.

Table 2 Layer-by-layer thermal conductivity of polystyrene concrete samples of different density.

\begin{tabular}{|c|c|c|c|}
\hline \multirow{2}{*}{ Layer } & \multicolumn{3}{|c|}{ Thermal conductivity $(\mathrm{W} / \mathrm{m} \cdot \mathrm{K})$} \\
\cline { 2 - 4 } & $\begin{array}{c}\text { Sample 1 } \\
(\text { Electro heating, } \\
\left.1200 \mathrm{~kg} / \mathrm{m}^{3}\right)\end{array}$ & $\begin{array}{c}\text { Sample 2 } \\
(\text { Electro heating, } \\
700 \mathrm{~kg} / \mathrm{m}^{3} \text { ) }\end{array}$ & $\begin{array}{c}\text { Sample 3 } \\
(\text { Standard } \\
\text { technology, 1200 } \\
\left.\mathrm{kg} / \mathrm{m}^{3}\right)\end{array}$ \\
\hline 1 layer & 0.2007 & 0.1283 & 0.2136 \\
\hline 2 layer & 0.1961 & 0.1274 & 0.2090 \\
\hline 3 layer & 0.1944 & 0.1268 & 0.2012 \\
\hline 4 layer & 0.1933 & 0.1261 & 0.1878 \\
\hline Segregation & $3.68 \%$ & $1.56 \%$ & $12.08 \%$ \\
\hline
\end{tabular}

The non-uniformity of the sample made according to the proposed technology is $3.68 \%$ against $12.08 \%$ of the sample made according to the standard technology.

The significant difference in thermal conductivity of the upper and lower layers of the polystyrene concrete block manufactured according to conventional technology is due to the flotation of the foamed polystyrene granules and sedimentation of the mortar part.

\section{Conclusion}

Practically all additive technologies offered to the construction market propose the use the $3 \mathrm{D}$ printer to print retained form made of cement and sand mortar of sand, clay or foamed polyurethane.

Then the standard (manual) operations follow to reinforce, place concrete and insulate the future enclosure structure. It is known that in the complex of building structures concreting the labour consumption of formwork does not exceed $40 \%$.

Consequently, the 3D printer will take over only this part of the labor intensity. The rest has to be done with the hands of the workers.

The use of the $3 \mathrm{D}$ printer that prints a full-width polystyrene wall allows to: 
- form the working layer over the entire width of the wall simplifying the binder supply system;

- eliminate the mobility of binding material as soon as it leaves the working head and accelerate hardening due to high temperatures of heat treatment;

- provide self-consolidation of polystyrene concrete while forming the working layer by means of increasing the volume of granules during their bloating;

- ensure the uniform distribution of foamed polystyrene granules over the volume of the wall material, thus increasing its high-quality thermal resistance;

- guarantee the specified strength or heat insulation characteristics of the material of the erected wall.

\section{References}

1. Patent US7641461B2 Robotic systems for automated construction B29C64/106/

2. V.P. Grakhov, S.A. Mokhnachev, O.V. Borozdov . Fundamental researches, 11-12, 2673-2676 (2014)

3. S.A. Udodov, ICSC Science and Enlightment 58-61 (Penza, 2017)

4. D.A. Luneva, E.O. Kozhevnikova, S.V. Kaloshina, Civil Engineering and Aarchitecture, 8 (1), 90-101 (2017)

5. K.A. Rudyak, Yu.O. Chernyshev, Materials of International scientific and practical conference, 147-151 (Kazan, 2016)

6. Ye.Yu. Stepanova, G.V. Barsukov, Yu.S. Stepanov, Technical Science, 8-2, 243-249 (Tula: Tula State University, 2016)

7. Winsun Company. 3D housing design (Electronic resource URL:http:/www.yhbm.com/index.php? $\mathrm{a}=$ lists\&c=index\&catid=67\&m=content) (date 14.12.2016).

8. Construction 3D: Nikita Chen-yun-tya was the first who printed the house in Russia. (Electronic resource https:/www.rbc.ru/magazine/2017/06/592567559a7947e1bb4b7ea9)

9. French project BATIPRINT for 3D house construction "inside out" (Electronic resource - URL:http://www3dprintingindustry.com/tags/ls2n/)

10. Institute for advanced architecture of catalonia takes a natural approach to on site $3 \mathrm{~d}$ printing (Electronic resource - URL: http://www 3dprintingindustry.com/tags/terraperforma)

11. The Method and the Device (Electronic resource - URL: http://www dshape.com/what-is-it/the-process/the-concept/) 